\title{
Exploration and Research on the Propagation Law of Seepage Risk Network in Tailings Storage Facility
}

\author{
Zhixin Zhen $\mathbb{D D}^{1}{ }^{1}$ Xuewei Ma ${ }^{(D)}{ }^{2}$ and Bo $\mathrm{Ma}^{1}$ \\ ${ }^{1}$ School of Civil and Resource Engineering, University of Science and Technology Beijing, Beijing, China \\ ${ }^{2}$ School of Finance and Taxation, Inner Mongolia University of Finance and Economics, Hohhot, China \\ Correspondence should be addressed to Zhixin Zhen; b20160029@xs.ustb.edu.cn
}

Received 24 July 2021; Accepted 13 October 2021; Published 8 November 2021

Academic Editor: Florentino Borondo

Copyright (c) 2021 Zhixin Zhen et al. This is an open access article distributed under the Creative Commons Attribution License, which permits unrestricted use, distribution, and reproduction in any medium, provided the original work is properly cited.

The seepage accident of a tailings pond poses a serious threat to the stability of tailings dams and the surrounding environment. To reduce the occurrence of seepage accidents, this paper studies the identification of seepage hazards, the propagation law of seepage risk, the importance of hazards, and the priority of hazard treatment. To overcome the subjectivity and omission of hazard identification, according to the complexity and dynamics of tailings seepage, this paper proposes the evidence-based identification method of three-dimensional seepage hazards (EIMTSH) to identify the hazards of the tailings seepage system and the relationship between hazards. Then, on the basis of identifying the hazards of the tailings seepage system, the propagation network of seepage risk in tailing ponds (PNSRTP) is constructed based on the complex network theory. By analyzing the characteristics of the PNSRTP, it can be found that the propagation of seepage risk is scale-free and small-world. Through the node deletion method, this paper finds that the nodes with a higher degree value can reduce the network efficiency more quickly and should be governed first. By giving priority to the treatment of hazards with higher degree, the propagation of seepage risk can be reduced more quickly and the risk management level of tailings ponds can be improved, which is helpful to realize the sustainable development of mining production.

\section{Introduction}

Tailings pond is a kind of geotechnical facility used for storing mine waste, which is called tailings in the mining industry. The composition of tailings is very complex, which may show strong corrosive, volatile, acidic, and other characteristics affected by the types of minerals mined. If the tailings cannot be managed effectively, the tailings may leak under the action of seepage, mainly in the form of flowing soil, piping, contact erosion, contact flowing soil, etc., which is called tailings seepage [1]. Seepage accident of tailings pond is one of the typical accident types of tailings pond, which not only directly pollutes the surrounding environment but also destroys the stability of the tailings dams. In serious cases, it will lead to dam breaks, causing greater accidents and ecological disasters $[2,3]$.

Seepage refers to the flow of fluid in porous media. The material which is composed of granular or fragmentary material and contains many pores or fissures is called the porous medium. Seepage is widely used in many fields. Tong Shujiao et al. used the two-dimensional seepage accident consequence analysis software to analyze the temporal and spatial variation law of leakage poison concentration [4]. In order to improve the design, management, and follow-up restoration of the landfill site, Shu et al. proposed a new simplified method to calculate breakthrough time of municipal solid waste landfill liners [5]. He et al. studied the biological damage to Sprague-Dawley rats by contaminated groundwater from rare earth metals tailings pond seepage at the individual, organ, tissue, and cell level [6]. CA LópezMorales and Lilia conducted research on seepage in wastewater treatment and reuse processes [7].

In order to correctly analyze the change law of seepage field and stress field caused by tailings storage and rainfall, we should attach great importance to the identification of seepage hazards. Hazard identification, also known as risk 
identification, is an important basis for risk management [8]. To achieve the sustainable development goals, Li used the official data of the Belt and Road Initiative (BRI) countries from year 2000 to year 2015 to identify socioeconomic vulnerability to natural hazards of Belt and Road Initiative countries [9]. Makowski and Niedbalski used the geomechanical method to identify rock burst hazards in underground mining, which helped to predict rock burst accidents before mining operations [10]. Ibrahim used the national data of fire anomalies to analyze the risk of wastefires in Sweden and found that controlling upstream hazards in waste management chain helped to reduce the risk of fire [11]. Ferreira et al. proposed a hazard classification system based on the use of the CFs of the virtual substances as a hazardous reference to help perform a preliminary screening, which can be integrated with other criteria to facilitate the identification of PBT chemicals [12]. Wu Deng et al. improved the safety level of complex systems by optimizing complex systems [13-15].

For the seepage problem of the dam body, there are dozens of common identification methods for hazards, such as fault type and impact analysis, prehazard analysis, checklist method, hazard and operability research, fault tree analysis, and event tree analysis [16]. In addition, some new methods are also applied to the identification of dam hazard. Gao Shipei et al. combined the detection of levee engineering with numerical simulation analysis, so as to determine the location, outline, and size of levee hazard [17]. Based on the distributed optical fiber temperature measurement technology, Wang monitored the leakage volume of homogeneous earth dam and the damage degree of optical fiber geomembrane, so as to realize a more comprehensive monitoring of the hazard of seepage field [18]. Ma et al. have conducted treatment and research on the leakage hazard of Yuecheng dam in combination with the engineering practice [19].

The cause of seepage accident in tailings pond is complex, and many influencing factors are coupled with each other. At the same time, the seepage system is constantly changing with the construction of tailings pond, which creates a big problem for the characterization of seepage risk propagation process in tailings pond. Complex network has the characteristics of node diversity and connection diversity, which can better represent the internal relationship between research objects (nodes) [20]. In order to promote the sustainable use of rare earths (RE), Xibo Wang built an embodied RE network by combining both input-output analysis and complex network theory [21]. Jiujie Shi used thw complex network analysis method to analyze the historical evolution of the international plastic wastes trade and showed that China's management policies are the main driving forces for the expansion and shrinkage of the global plastic wastes trade network [22]. Guo S took the Chinese construction industry as an example and conducted research and exploration on the accident behavior risk chain network based on the accident causality theory [23]. Mohmand and Wang studied the structural properties of the Pakistan railway network (PRN) [24]. After the construction of the complex network which represents the relationship between nodes, scholars began to try to use the characteristics of the complex network to find the key nodes that dominate the operation of the network. Yu et al. converted the critical node identification problem in complex networks into a regression problem and presented it to identify critical nodes with the best spreading ability [25]. Because it is difficult to distinguish the importance of nodes with the same degree, an average shortest path centrality to rank the spreaders was proposed [26]. Zhen et al. initially tried to apply the important nodes of complex network identified by the network centrality index to the prevention of tailing pond accidents [27].

\section{Research Overview}

In order to explore and study the propagation law of seepage risk in tailings storage facility, this paper studies this problem in three steps. Firstly, based on the constitution and function of the seepage system of tailings ponds, the hazards in different life cycle stages of the tailings pond are identified objectively and systematically by using laws and regulations, literature, and accident cases, and the list of seepage hazards and propagation paths of seepage risk are determined after the same hazards are integrated. Secondly, based on the list of seepage hazards, this paper uses the Pajek software to construct the propagation network of seepage risk in tailing ponds (PNSRTP) for the first time; Finally, this paper analyzes the characteristics of this PNSRTP with the help of some analysis methods in the complex network theory and tries to find the key hazards that dominate the propagation of seepage risk from the perspective of reducing the network efficiency. The entire research process of this paper is shown in Figure 1.

\section{Identification of Seepage Hazard in Tailings Pond}

3.1. Identification Method. A hazard may cause loss of life, injury, or other health impacts, property damage, social and economic disruption, or environmental degradation [28]. In this paper, according to the characteristics of the tailing pond seepage system with the dynamic change of tailing pond life cycle, combined with industry laws and regulations and technical specifications, the seepage hazards of tailing ponds are divided into three modes or three states from the perspective of safety production. The first state is the dormant hazard, which is the initial factor or event to cause the seepage. It is an indirect factor and cannot be triggered by other factors or events. Its state is basically stable. The second is the armed hazard, which refers to the intermediate state of hazard evolution. It may evolve from the dormant hazard, or other armed hazards may cause it [29]. The third is the active hazard, which mainly refers to the seepage events that are happening [29].

The causes of seepage accidents in tailings pond are complex, and the seepage system changes continuously with the life cycle of tailings pond. These characteristics make it difficult to identify the seepage hazards in tailings ponds. Although some scholars have proposed a series of hazard 




Figure 1: The flow chart of research methods and results.

identification methods for such dynamic complex systems, these methods are mainly based on the experience and subjective judgment of the identification personnel. The result is that some key hazards may be missed [30]. In order to overcome the above shortcomings, this paper proposes a method to identify the seepage hazards of tailings pond, which is called the evidence-based identification method of three-dimensional seepage hazards (EIMTSH), as shown in Figure 2.

It can be seen from Figure 2 that the EIMTSH includes three dimensions and each point in the space specifically represents the seepage hazards and influencing factors at each life cycle stage of tailings ponds under different supporting evidence. The $Y$-axis is composed of eight subsystems of the tailings pond seepage system, mainly including pond area subsystem, personnel subsystem, conveyor subsystem, drainage subsystem, monitoring subsystem, dam subsystem, material subsystem, and management subsystem. Each subsystem can be divided into different modules according to its function and structural characteristics. The $Z$-axis is the life cycle stage of tailings pond, which mainly includes construction stage, operation stage, closing stage, and reclamation stage or recovery stage. The division method of $Z$-axis and $Y$-axis is used to divide all the objects that need to be identified at different time periods and in different subsystems [29]. The $X$-axis is the supporting evidence used to identify the potential seepage hazards of tailings ponds, mainly including the relevant technical specifications and laws and regulations, seepage accident cases, literature, news media information, etc., as shown in Appendix A, B, and C [29].

In order to find out all the seepage hazards, the paper analyzes the Code for Design of Tailings Facilities (GB 50863-2013), Code for Construction and Acceptance of Tailings Facilities (GB-T 50864-2013), and other evidence in Appendix A and then extract the hazards and the influence mechanism between hazards in each content one by one [29]. At the same time, referring to the content in Appendix $\mathrm{B}$ and Appendix $\mathrm{C}$, we can get all the hazards of the seepage system in tailings ponds that has not been integrated, that is, all the spatial nodes in Figure 2. After integrating these identified hazards, all hazards of the seepage system in tailings ponds can be obtained, as shown in Table 1.

3.2. Identification Result. Based on the EIMTSH, the seepage hazards of tailings pond are systematically identified. In this paper, 313 seepage hazards of tailings ponds are identified and 1912 relationships among them are shown in Table 1
[31]. In the first column of Table 1, the names of eight subsystems of seepage system of tailings pond are listed. The fourth column is the hazard identified by the EIMTSH, which is given as the number in the third column. The second column indicates the specific category of the seepage subsystem of tailings ponds to which the fourth column of hazards belongs. The fifth column indicates that the hazard in the fourth column of the same row is identified by the supporting evidence, and the type and location of the evidence are marked. The details of the evidence are shown in Appendix. The last column is the hazard number, which indicates the hazard or event directly caused by the hazard in the fourth column of the same row.

Take hazard 77 (The tailings dam slope ratio is unreasonable) as an example, the number of this hazard is 77 , which belongs to the dam body class of the dam system. Based on the evidence F1-4.5, W4, and W7, we can confirm the existence of hazard 77 and find that hazard 77 can directly cause hazards 62, 64, 65, 73, and 157 . Evidence F represents laws, standards, and norms, and $\mathrm{W}$ represents the scientific literature and accurate case. F1-4.1 indicates that the evidence is located in the first section of Chapter IV of 'Code for Design of Tailings Facilities (GB 50863-2013)'. More detailed supporting evidence information is provided in appendix $\mathrm{A}, \mathrm{B}$, and $\mathrm{C}$.

\section{Propagation Network of Seepage Risk in Tailings Pond}

4.1. Model Construction. In order to apply the complex network model to characterize the seepage risk evolution process of tailings pond, this paper uses the seepage hazard to represent the network node and the relationship between hazards represents the network edge. At the same time, according to the three states of seepage hazards mentioned above, the PNSRTP is divided into three-layer nodes (dormant hazard, threat hazard, and activity hazard/accident) and two stages (from dormant hazard to threat hazard and from threat hazard to activity hazard). In this paper, with the help of complex network software Pajek, the hazard and the relationship between hazards in Table 1 are constructed as the PNSRTP, as shown in Figure 3.

In Figure 3, the first layer of nodes constitutes dormant hazards, represented by yellow nodes, with a total of 31 . Dormant hazards are the initial hazards that cause other hazards, which cannot be caused by others. Earthquakes, floods, strong winds, etc., are all dormant hazards. The second layer of nodes includes armed hazards, represented 




FIgURE 2: The evidence-based identification method of three-dimensional seepage hazards.

TABLE 1: List of seepage hazards in tailings pond.




TABle 1: Continued.

\begin{tabular}{|c|c|c|c|c|c|}
\hline $\begin{array}{l}\text { Talings dam } \\
\text { subsytem }\end{array}$ & Modules of the subsystem & $\begin{array}{c}\text { Number } \\
(v)\end{array}$ & Haard name & Evidence & Number of hazards caused \\
\hline & & $\begin{array}{c}63 \\
65\end{array}$ & $\begin{array}{l}\text { Decrecase of dam clevation } \\
\text { Dam medromation }\end{array}$ & W6, W11, W6, C2 & $39,190,194$ \\
\hline & & $\begin{array}{l}65 \\
66\end{array}$ & 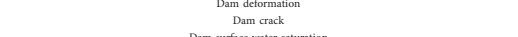 &  & 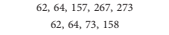 \\
\hline & & $\begin{array}{c}67 \\
68\end{array}$ & 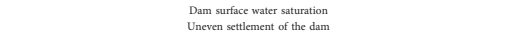 &  & 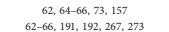 \\
\hline & & $\begin{array}{l}69 \\
69 \\
70\end{array}$ & 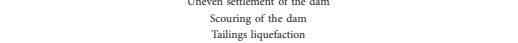 & 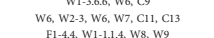 & 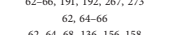 \\
\hline & & $\frac{11}{71}$ & 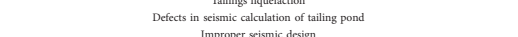 & 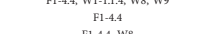 & 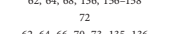 \\
\hline & & $\begin{array}{c}72 \\
75 \\
77\end{array}$ & 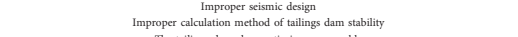 & 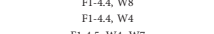 & 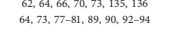 \\
\hline & & ${ }_{78}^{77}$ & 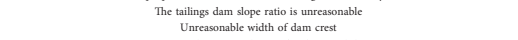 & 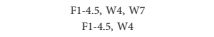 & $62,646,5,73,157$ \\
\hline & & ${ }_{80}^{79}$ & $\begin{array}{l}\text { Improper dam type slection for the initia dam } \\
\text { The he hight of initial dam is unrecasonalle }\end{array}$ & $\begin{array}{c}\mathrm{W}_{1-1.1 .2} \\
\mathrm{~F} 1-41, \mathrm{w}, \mathrm{W}\end{array}$ & 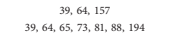 \\
\hline & Dam body & $\begin{array}{c}81 \\
82\end{array}$ & 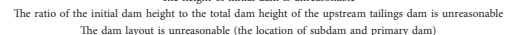 & 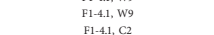 & 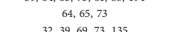 \\
\hline & & $\begin{array}{ccc}62 \\
61 \\
83\end{array}$ & 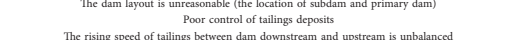 & w1-1.1.3 & $64,65,68,77,152,154,155,157$ \\
\hline & & $\begin{array}{l}83 \\
84\end{array}$ & 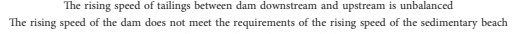 & 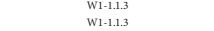 & ${ }_{80,61}^{61} 85.86$ \\
\hline & & $\begin{array}{c}85 \\
86\end{array}$ & 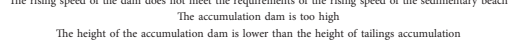 & F1-4.1, F5 & 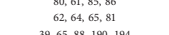 \\
\hline & & $\begin{array}{l}80 \\
87\end{array}$ &  & 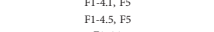 & 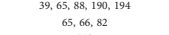 \\
\hline & & $\begin{array}{c}88 \\
89\end{array}$ & 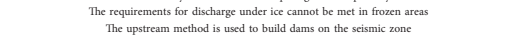 & 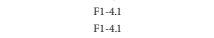 & $\begin{array}{c}112 \\
60,62,70\end{array}$ \\
\hline & & $\underset{90}{90}$ & 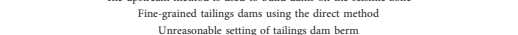 & $\begin{array}{ll}\mathrm{F} 1.41 \\
\mathrm{~F} 1.45\end{array}$ & 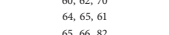 \\
\hline & & $\begin{array}{c}91 \\
92\end{array}$ & 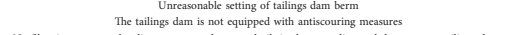 & 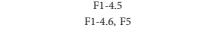 & $\begin{array}{l}65.68 .82 \\
6982\end{array}$ \\
\hline & & ${ }_{93}^{93}$ &  & 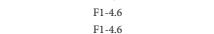 & $\begin{array}{c}64,82 \\
34,64.65 \\
3.03\end{array}$ \\
\hline & & $\begin{array}{c}95 \\
96 \\
96\end{array}$ & 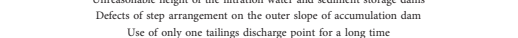 & 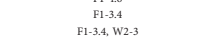 & $\begin{array}{l}65.60,82 \\
61.685\end{array}$ \\
\hline & & 97 & 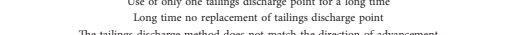 & F1-3.3, W2-3 & 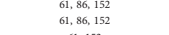 \\
\hline & & $\begin{array}{c}98 \\
99 \\
99\end{array}$ & 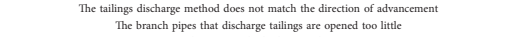 & 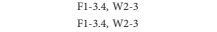 & $\begin{array}{c}61.152 \\
61,152,234\end{array}$ \\
\hline & & $\begin{array}{l}100 \\
101\end{array}$ & 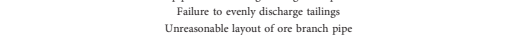 & $\begin{array}{c}\mathrm{F} 1-3.4, \mathrm{~W}_{2}-3 \\
\mathrm{~F}-134\end{array}$ & 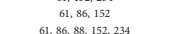 \\
\hline & & $\begin{array}{l}700 \\
102\end{array}$ & 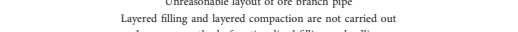 & 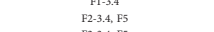 & 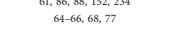 \\
\hline & & $\begin{array}{l}103 \\
104\end{array}$ & 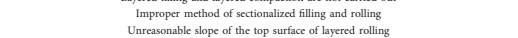 & 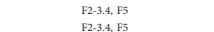 & 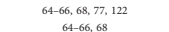 \\
\hline & & $\begin{array}{l}105 \\
106\end{array}$ & 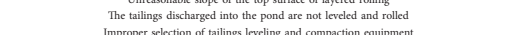 & 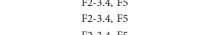 & $\begin{array}{c}64-66,68 \\
86\end{array}$ \\
\hline & & $\begin{array}{l}106 \\
107\end{array}$ &  & 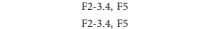 &  \\
\hline & & ${ }_{108}^{108}$ &  &  & $\begin{array}{l}65,66,104 \\
65,66,68,104,122,158\end{array}$ \\
\hline & & ${ }_{109}^{109}$ &  & F2.3.4, 55 & $\begin{array}{l}-125 \\
65,66,68,104,122\end{array}$ \\
\hline & & $\begin{array}{l}1100 \\
111\end{array}$ & $\begin{array}{l}\text { Improper paring } \\
\text { Downhill paving when hit ground is unerenen }\end{array}$ &  & 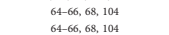 \\
\hline & Dam flling & 112 &  & F-3.3., , F5 &  \\
\hline & & $\begin{array}{l}113 \\
114\end{array}$ &  &  & $\begin{array}{l}65,66 \\
646,77\end{array}$ \\
\hline & & $\begin{array}{c}115 \\
116\end{array}$ &  &  & $\begin{array}{c}64,6,67 \\
65,66\end{array}$ \\
\hline & & $\begin{array}{l}1116 \\
117\end{array}$ &  &  & $\begin{array}{ll}112 \\
65,66,73,122\end{array}$ \\
\hline & & $\begin{array}{c}118 \\
120\end{array}$ &  &  & $\begin{array}{c}64,77,78 \\
65618\end{array}$ \\
\hline & & ${ }_{121}^{121}$ &  & $w_{1} \cdot 3.44$ & $\begin{array}{l}65,66,1,138 \\
65,658\end{array}$ \\
\hline & & ${ }_{122}^{122}$ &  &  & $\begin{array}{l}64,66,73 \\
19665\end{array}$ \\
\hline & & $\begin{array}{l}124 \\
125\end{array}$ &  & 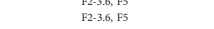 & $19,62,64,65,73,122$ \\
\hline & & ${ }_{126}$ &  & ${ }^{\mathrm{F} 23.3 .6}$ & $19,62,64-66,73,77,122,157$ \\
\hline & & 127 &  & F1.5 & 64,67 \\
\hline & & 128 &  & F1.5 & $64,65,68$ \\
\hline & & $\begin{array}{l}129 \\
131\end{array}$ & 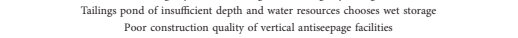 & $\begin{array}{c}\mathrm{F} 1.5 \\
\mathrm{~F} 2.3 .2\end{array}$ & $\begin{array}{c}39.165 \\
66.157 .165\end{array}$ \\
\hline & & 132 & 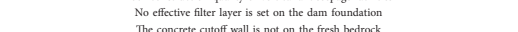 & w1-3.4.4. & $\begin{array}{l}6,151,765 \\
131,157,165\end{array}$ \\
\hline & & $\begin{array}{l}133 \\
134\end{array}$ & 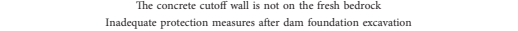 &  & $131,157,165$ \\
\hline \multirow{48}{*}{ Dam subsystem } & & $\begin{array}{l}154 \\
135\end{array}$ & 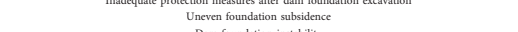 & 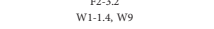 & $\begin{array}{l}66,131,135,136 \\
63-66,68,73,136,191,267,273\end{array}$ \\
\hline & Dam foundation & 136 & Dam foundition instablity & F4, FG, W1-1.4, W11, W12, ws, w9 & 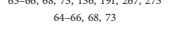 \\
\hline & & ${ }^{137}$ & 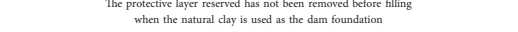 & F2:32. F5 & 131, 133, 135, 136, 158 \\
\hline & & 138 &  & F2-3.3., F5 & $131,133,135,136,165$ \\
\hline & & $\begin{array}{l}139 \\
140\end{array}$ &  & $\begin{array}{l}\mathrm{F} 2-3.2,25 \\
\mathrm{~F} 2-3.255\end{array}$ & $\begin{array}{l}131,133,135,136,158 \\
1331,133,135,136,158\end{array}$ \\
\hline & & ${ }_{141}^{141}$ &  & $\begin{array}{l}\text { F2-3.2., F5 } \\
w_{1-1344}\end{array}$ & \\
\hline & & $\begin{array}{l}145 \\
146\end{array}$ &  & F1-5.5.7. & ${ }_{53}^{53,157}$ \\
\hline & & 147 &  & $W_{1-3.34,4,41-5,7}$ & 53 \\
\hline & & $\begin{array}{l}148 \\
130\end{array}$ & 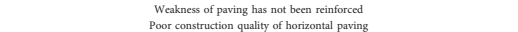 & $\begin{array}{c}\mathrm{W}_{1-3.34,4, \mathrm{F5}} \\
\mathrm{W}_{1}-3,44\end{array}$ & 158 \\
\hline & Dry beach & $\begin{array}{l}130 \\
149\end{array}$ &  &  & 1.157 \\
\hline & & $\begin{array}{l}150 \\
151\end{array}$ & 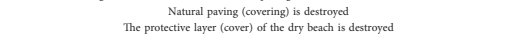 & $\begin{array}{c}\mathrm{W}_{1-3.44} \\
\mathrm{w}_{1-3.44}\end{array}$ & $\begin{array}{c}158 \\
53.158\end{array}$ \\
\hline & & ${ }_{152}^{152}$ &  & 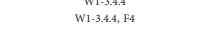 & $\begin{array}{l}533,158 \\
154,157\end{array}$ \\
\hline & & $\begin{array}{l}154 \\
155\end{array}$ & 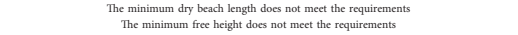 & $\begin{array}{l}\text { Fil-42, ws } \\
\text { fllt2 ws }\end{array}$ & 64,167 \\
\hline & & 156 & 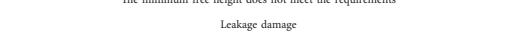 &  & $\begin{array}{c}64,167 \\
60,62.64\end{array}$ \\
\hline & & 157 & Filter filure & $\underset{\text { Col.1.13 }}{\text { W1.34, w8 }}$ & $\begin{array}{l}60.60,64 \\
64,67,136,156,167,195\end{array}$ \\
\hline & & 158 & 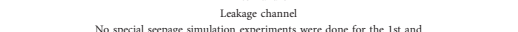 & W1.34, ws, C1, C5, C9.C10, C12-C13 &  \\
\hline & & 159 & 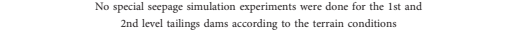 & Fl-43 & $93,162,167$ \\
\hline & & 160 & The secpage calculation of the dam body was not tarriced out in the preclininary decign stage & Flit3 & $93,162,167$ \\
\hline & & $\begin{array}{l}161 \\
162\end{array}$ & 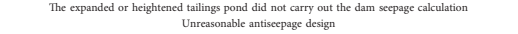 & 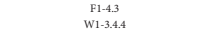 & $\begin{array}{c}162.167 \\
6.156-157\end{array}$ \\
\hline & & 164 & 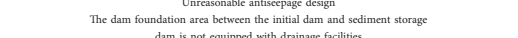 & $w_{1-3.44}$ & (157/78 \\
\hline & & 165 & 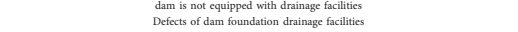 & $\mathrm{w}_{1.3,4}$ & 157 \\
\hline & & $\begin{array}{c}166 \\
167\end{array}$ & 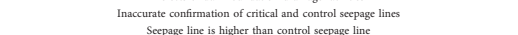 & 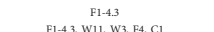 & $\frac{167}{65.615156}$ \\
\hline & & 168 & 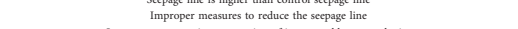 & 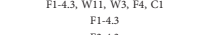 & $\begin{array}{l}65-67,757-156 \\
167\end{array}$ \\
\hline & & ${ }_{169}^{169}$ & 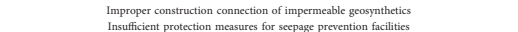 & F2-4.3. & $\begin{array}{l}157,165,184 \\
1.88\end{array}$ \\
\hline & & ${ }_{171}^{170}$ & 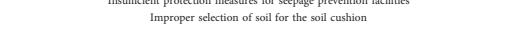 & 丝2-11 & $\begin{array}{l}158,165,183 \\
157,765,184\end{array}$ \\
\hline & & $\begin{array}{l}172 \\
173\end{array}$ & 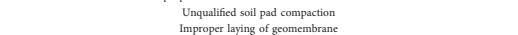 & $\begin{array}{c}\mathrm{F} 2-11 \\
\mathrm{~F}-21 \mathrm{H}\end{array}$ & $\begin{array}{l}1577.165 .184 \\
1.75\end{array}$ \\
\hline & & 365 & 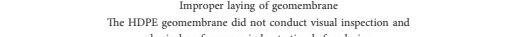 & 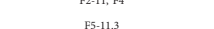 & $157,165,184$ \\
\hline & & 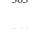 & 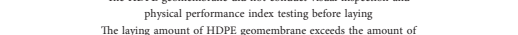 & F5.1.3. & ${ }^{173}$ \\
\hline & & 366 & 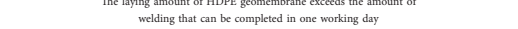 & Fs-11.3 & 173 \\
\hline & & $\begin{array}{l}367 \\
368\end{array}$ & 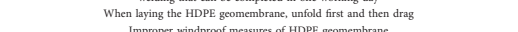 & F5-11.3. & 173 \\
\hline & Scepage & $\begin{array}{l}368 \\
369 \\
369\end{array}$ & 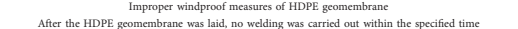 & $\begin{array}{l}\mathrm{F} 5 \mathrm{11.3}, \mathrm{F} \\
\mathrm{F} 5 \mathrm{1113}\end{array}$ & 1773 \\
\hline & & 370 & 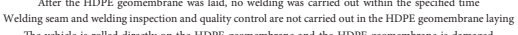 & F5-11.3. & 173 \\
\hline & & ${ }^{371}$ & & F5-11.3. & \\
\hline & & 372 & 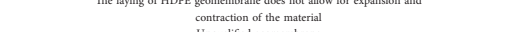 & F5-11.3. & 173 \\
\hline & & 174 & Unqualificed geomembrane & F2-11 & $157,165,184$ \\
\hline & & $\begin{array}{l}175 \\
176\end{array}$ & 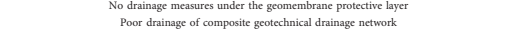 & $\begin{array}{l}\mathrm{F} 2 \cdot 1 \\
\mathrm{~F} 211\end{array}$ & $\begin{array}{l}157.165,185 \\
157,165\end{array}$ \\
\hline & & 177 & 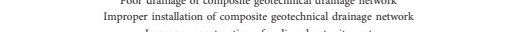 & F2-11 & $157,165,176,183$ \\
\hline & & $\begin{array}{ll}378 \\
3\end{array}$ & 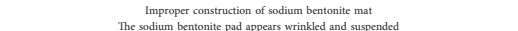 & $\begin{array}{c}\text { F2-11 } \\
\text { F-1.15 }\end{array}$ & $\begin{array}{l}157,165,184 \\
178\end{array}$ \\
\hline & & 361 & 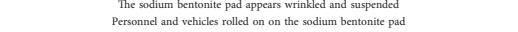 & F5-11.5 & $\begin{array}{l}778 \\
178\end{array}$ \\
\hline & & 362 & 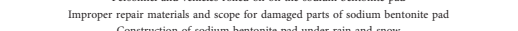 & F5-11.5 & 178 \\
\hline & & $\begin{array}{l}{ }_{363}^{363} \\
364\end{array}$ & 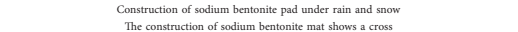 & & $\begin{array}{l}178 \\
178\end{array}$ \\
\hline & & 179 & 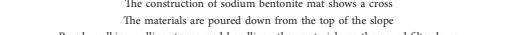 & F2:35 & 184 \\
\hline & & 180 & & F2.3.5. & 183 \\
\hline & & $\begin{array}{l}181 \\
182\end{array}$ & 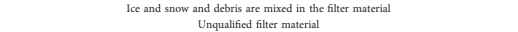 & $\begin{array}{l}\mathrm{F} 2.3 .5 \\
\mathrm{~F} 2.3 .5\end{array}$ & $\begin{array}{l}182 \\
183\end{array}$ \\
\hline & & 183 & 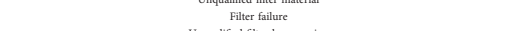 & $\mathrm{F} 2-3.5, \mathrm{~W}_{2}-3, \mathrm{~W} 3, \mathrm{~F} 4$ & $65.157,165$ \\
\hline & & $\begin{array}{l}184 \\
186 \\
186\end{array}$ & 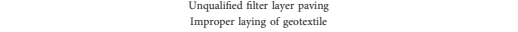 & 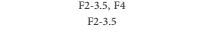 & $\begin{array}{c}66.138 \\
165.184\end{array}$ \\
\hline & & $\begin{array}{l}7180 \\
187\end{array}$ & 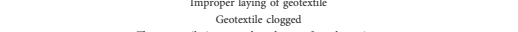 & 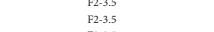 & $\begin{array}{l}105,1,184 \\
105,183\end{array}$ \\
\hline & & $\begin{array}{l}188 \\
189\end{array}$ &  & F.2.5. & 165, 1833 183 \\
\hline
\end{tabular}


TABle 1: Continued.

\begin{tabular}{|c|c|}
\hline $\begin{array}{l}\text { Tailings dam } \\
\text { subsystem }\end{array}$ & Modules of the subsystem \\
\hline & \\
\hline & \\
\hline & \\
\hline & \\
\hline & \\
\hline & \\
\hline & \\
\hline & \\
\hline & \\
\hline & \\
\hline & \\
\hline & \\
\hline & Drainage plan \\
\hline Drainage subsystem & \\
\hline & \\
\hline & \\
\hline & \\
\hline & \\
\hline & \\
\hline & \\
\hline & \\
\hline & \\
\hline & \\
\hline & \\
\hline & \\
\hline & Backwater plan \\
\hline & 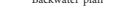 \\
\hline & \\
\hline & \\
\hline & \\
\hline & \\
\hline & \\
\hline & \\
\hline & \\
\hline & \\
\hline & \\
\hline & \\
\hline & \\
\hline & \\
\hline & \\
\hline & Convering facilities \\
\hline & \\
\hline & \\
\hline & \\
\hline & \\
\hline & \\
\hline & \\
\hline & \\
\hline & \\
\hline & \\
\hline & \\
\hline & \\
\hline & \\
\hline & \\
\hline & \\
\hline & \\
\hline & \\
\hline & \\
\hline & \\
\hline & \\
\hline & \\
\hline Conveyor subsystem & \\
\hline & Other transportation \\
\hline & facilities \\
\hline & \\
\hline & \\
\hline & \\
\hline & \\
\hline & \\
\hline & \\
\hline & \\
\hline & \\
\hline & \\
\hline & \\
\hline & \\
\hline & \\
\hline & \\
\hline & \\
\hline & \\
\hline & \\
\hline & \\
\hline & \\
\hline & \\
\hline & \\
\hline & Pump \\
\hline & \\
\hline & \\
\hline & \\
\hline & \\
\hline & \\
\hline & \\
\hline & \\
\hline & \\
\hline & \\
\hline & \\
\hline & \\
\hline
\end{tabular}


TABLE 1: Continued.

\begin{tabular}{|c|c|c|c|c|c|}
\hline $\begin{array}{l}\text { Talilings dam } \\
\text { subsystem }\end{array}$ & Modules of the subsystem & $\begin{array}{c}\text { Number } \\
(v)\end{array}$ & Hazard name & Evidence & Number of hazards caused \\
\hline \multirow{11}{*}{$\begin{array}{l}\text { Monitoring } \\
\text { subsystem }\end{array}$} & & 324 & 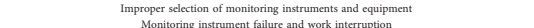 & F1-3.3, W8, F4, C2 & $\begin{array}{c}235,327,343 \\
3,37343\end{array}$ \\
\hline & & & 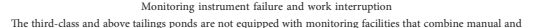 & F1-3.3, W8, C2 & 327,343 \\
\hline & & 326 & $\begin{array}{l}\text { The third-class and above talings ponds are not cquiped with monitoring facilities that combine manual and } \\
\text { automatic monitoring }\end{array}$ & $\mathrm{F} 1-3.4$ & $19,22-24,154,155,231,327$ \\
\hline & & 327 & Safety monitoring facilities cannot fully reflect the operating status of the tailings pond & F1-3.3., $\mathrm{C}_{2}$ & $7,19,22-24,37,45-50,65-69,135,136,191,192,200,267,343$ \\
\hline & & 328 & 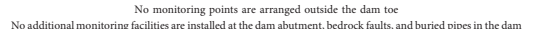 & $\mathrm{w}_{1-3.4 .5}$ & $19,22,23,231,310,327$ \\
\hline & & $\begin{array}{l}339 \\
330\end{array}$ & $\begin{array}{l}\text { No additional monitoring facilitics are installed a the dam abutment, becrock faults, and buried pipes in the dam } \\
\text { No monitoring of the amount and composition of talings entering the pond }\end{array}$ & $\mathrm{w}_{1-3.4 .5}$ & $65-69,135,136,191,192,267,327$ \\
\hline & & 331 & $\begin{array}{l}\text { No monitoring of fhe amount and compositito of taliligns estering the pond } \\
\text { No external drainge and composition monitoring }\end{array}$ & $\begin{array}{c}\mathrm{Fl}-3.3 .4 \\
\mathrm{~F} 1-3.4\end{array}$ & 45-50,327 \\
\hline & & & No monitoring of groundwater and surrounding water bodics & $\mathrm{F} 1-3.4 \mathrm{~A}$ & $\begin{array}{l}200,327 \\
327\end{array}$ \\
\hline & & 334 & The number of water quality monitoring wells around the tailings pond is insufficient & $\mathrm{F} 1-3.4 \mathrm{~s}$ & ${ }_{327}^{2327}$ \\
\hline & & 336 & The setting of monitoring facilitites is not included in the construction plan & F2-3.7. & 325,327 \\
\hline & & 337 & Improper safety monitoring during tunnel excavation & F2-6.1 & $214,271,273-276,289,327$ \\
\hline \multirow{10}{*}{$\begin{array}{l}\text { Management } \\
\text { subsystem }\end{array}$} & & 339 & Insufficient capital investment & $\mathrm{w}_{2}-3, \mathrm{~W}_{3}$ & 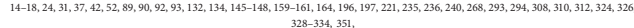 \\
\hline & & $\begin{array}{l}340 \\
343\end{array}$ & $\begin{array}{l}\text { Insufficient safety supervision } \\
\text { Inadequate saftety evaluation }\end{array}$ & $\begin{array}{l}\mathrm{W}_{2-3}-\mathrm{W}_{11}, \mathrm{~W}_{12}, \mathrm{~W} 5, \mathrm{CA}_{4} \\
\mathrm{~W}_{2}-3, \mathrm{~W}_{3}, \mathrm{~W}_{5}\end{array}$ & $\begin{array}{r}7,14-19,22-24,26,31,36,37,42-52,60,62-70,73,74,76-1737,1974178,179-214,218-298,299,307,308-337,346,348,351-355 \\
19,31,60,156,190,200,224,231,327\end{array}$ \\
\hline & & 344 & Outdated specifications and standards for survey, design, construction, and acceptance & $\mathrm{w}_{2-3, \mathrm{w} 3}$ & 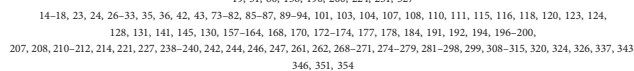 \\
\hline & & 345 & Defects in saftety production rules and regulations and operating procedures & $\mathrm{w}_{2-3,3} \mathrm{~W}_{3}$ & 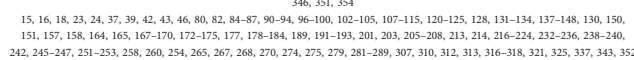 \\
\hline & & 346 & Improper data management & F2-1, F2-3.7. & 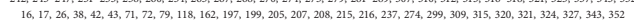 \\
\hline & & 347 & Insufficient or wrong hydrological and geological data & ${ }_{\mathrm{F} 2-1}$ & $16,17,26,38,42,43,71,72,79,118,162,197,199,205,207,208,215,216,237,274,299,309,315,320,321,324,327,343,352$ \\
\hline & & $\begin{array}{llll}348 \\
351\end{array}$ & 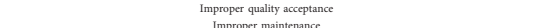 & 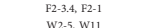 & $19,60,62-70,135,136,156-158,167,183,190-193,200,231,232,234,238,239,253,267,307,310,312,313$ \\
\hline & & $\begin{array}{l}335 \\
352\end{array}$ & $\begin{array}{l}\text { IIpproper ramintenance } \\
\text { Design defects of emergency plan }\end{array}$ & $\begin{array}{l}\text { W2.5, W11 } \\
\text { W2.5. W8 }\end{array}$ & 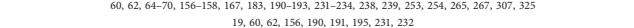 \\
\hline & & 354 & $\begin{array}{l}\text { Insufficient emergency plan drills } \\
\text {. }\end{array}$ & w2.5, ws & $\begin{array}{l}1,960,62,1,165,199,191,195,2131,232 \\
19,60,62,156,190,191,195,231,232\end{array}$ \\
\hline & & 355 & Insufficient experience in personnel or organization qualification problems & F2-1, W8, W11, W2-3 & 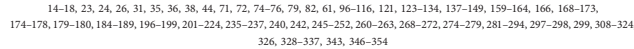 \\
\hline
\end{tabular}

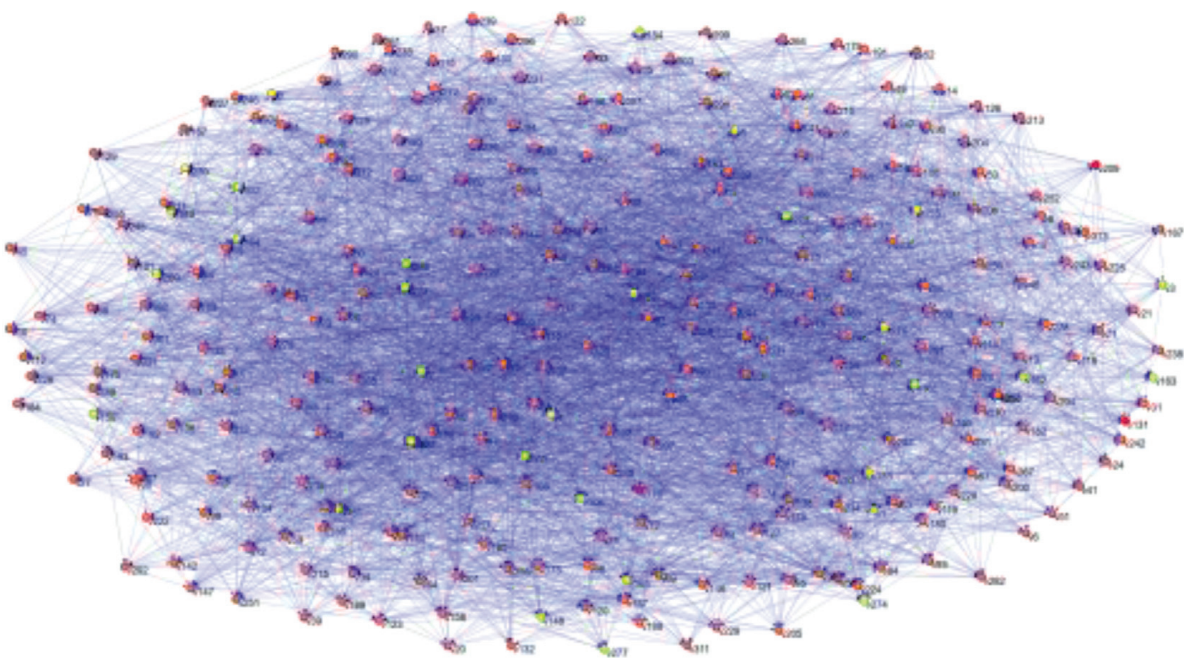

Figure 3: Model of the PNSRTP.

by orange, with a total of 279 . The second-layer node can be formed by the evolution of the initial dormant hazard and can also be caused by other armed hazards. The evolution relationship between hazards is complex, such as slope ratio, rapid rise of pond water level, and high phreatic line. The third-layer nodes are active hazards, which are indicated by red nodes, indicating that the accidents are happening or have happened. The seepage system of the tailings pond mainly includes three types of accidents: overtopping, leaks in transmission facilities, and seepage damage.

\subsection{Analysis of Network Statistical Characteristics}

4.2.1. Degree Values and Degree Distribution. The degree values of each node in the PNSRTP are shown in Figure 4. The degree value of the seepage hazard node is an important index to reflect the influence of hazards. According to the different directions of the relationship between hazards, it can be divided into out degree and in degree. Through the calculation using Pajek software, the average degree of the PNSRTP is found to be 12.22 and the network density is 0.02 . It shows that a node with potential seepage hazard is directly related to 12.22 hazards on average, but the overall density of the PNSRTP is not large.

As can be seen from Figure 4, the node with the largest degree is 340 (insufficient safety supervision), which can directly affect 261 kinds of hazards. 355 (lack of qualification and experience of personnel and institutions) belongs to the personnel system, which is the second largest hazard (182). The degree values of 345 (defects of safety production rules and regulations and operation procedures) and 344 (outdated specifications and standards for survey, design, construction, and acceptance) are 145 and 136, respectively. The degree values of 65 (dam deformation), 66 (dam crack), and 191 (drainage structure fracture) are all 59, of which 65 and 66 belong to the dam system and 191 belongs to the drainage system. 157 (filter water failure) also belongs to the dam system, for which the degree value is 49 . The degree value of 339 (insufficient capital input) is 45 , which belongs to the 


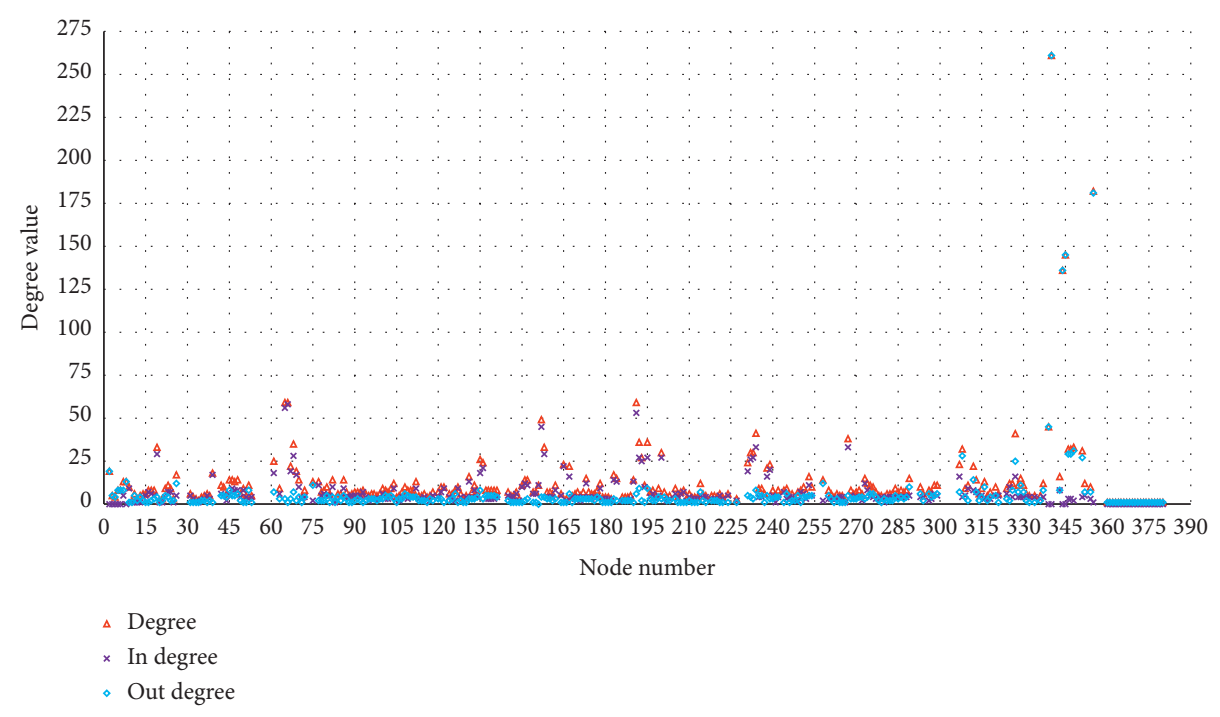

Figure 4: Node degree in the PNSRTP.

management system with hazards 340,344 , and 345 , which highlights that the management factor plays a vital role in the safety management of the tailings seepage system. The degree value of 234 (blockage or siltation) is 41 , which is the smallest hazard in the top ten hazards and also the only hazard belonging to the conveying system. It shows that the conveying system also has a certain impact on the seepage of tailings pond.

From the perspective of out degree, 340 (lack of safety supervision), 355 (lack of qualification and experience of personnel and institutions), 345 (defects in safety production rules and regulations and operating procedures), 344 (outdated specifications and standards for survey, design, construction, and acceptance), and 339 (insufficient capital investment) are the five biggest hazards, which are 261, 181, 145,136 , and 45 , respectively. These hazards can easily trigger other hazards, which are important causes of seepage risk transmission. 65 (dam deformation), 66 (dam crack), 191 (drainage structure fracture), 157 (filter water failure), and 234 (blockage or siltation) are the five biggest hazards of in-degree value, which have degree values $58,56,53,45$, and 33 respectively. Because these are easily caused by other hazards, these hazards should be monitored and paid attention to in the prevention of seepage accidents.

The cumulative degree distribution of the PNSRTP is shown in Figure 5. The cumulative degree distribution presents a power-law distribution that has the approximate fit $P(k)=3.8428 x^{-1.192}\left(R^{2}=0.9468\right)[23]$. The above result deviates from the power-law nature for lager $k$, which indicates that the PNSRTP has scale-free property [23]. The scale-free property shows that a few nodes have high degree values in the PNSRTP, which is consistent with the distribution of degree values in Figure 4 . These nodes make the PNSRTP robust to random attacks. This is reflected in the fact that although the world's major economies have invested a lot of resources in the safety management of the seepage system of tailings pond in the past, the seepage accidents of tailings pond often occur due to the inability to

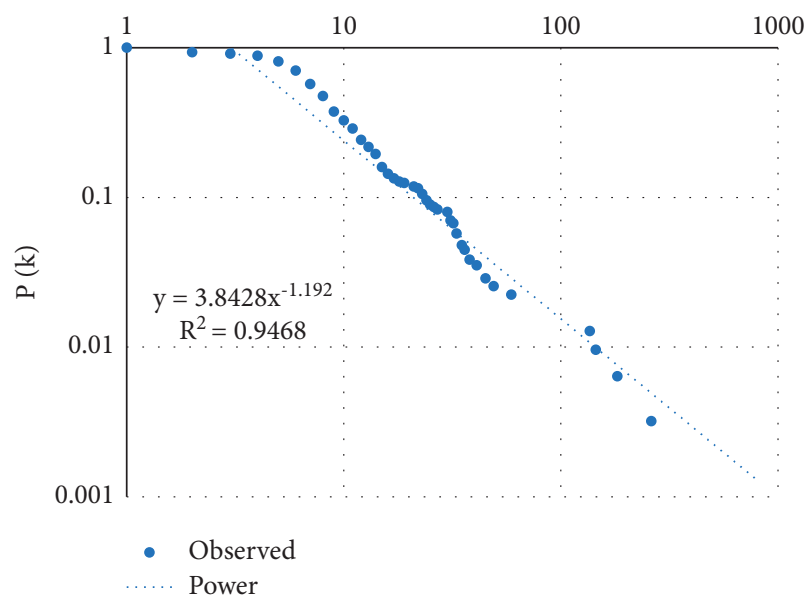

FIgUre 5: Cumulative degree distribution of the PNSRTP.

find out the key hazards (determine the importance of the hazards). At the same time, these high degree value nodes show vulnerability to specific attacks. If we can apply this characteristic to the treatment of seepage risk of tailings pond, determine the optimal treatment sequence of hazards, and block the correlation between hazards, the occurrence of seepage accidents will be greatly reduced.

\subsubsection{Average Path Length and Network Diameter.} According to Pajek calculation, the diameter of the PNSRT network is 13, which represents the longest distance between two hazard nodes in the network. The distance between node 9 (mudslide) and 224 (low tailings water recovery rate) or node 8 (beyond standard earthquake) and 207 (flood drainage structures are directly located on the tailings sediment beach) is 13 . They are the node pairs with the farthest risk propagation distance in the network.

The average path length of the PNSRTP is 4.90, which means that the seepage risk propagates from one hazard 
node to other hazard nodes in the network with an average of no more than 5 nodes. The PNSRTP has more than 300 hazard nodes and nearly 2000 hazard relationships, but the average distance between the two hazard nodes is very short. The above characteristics show that the cause of seepage risk is complex and the propagation speed is fast. When the seepage risk is formed, if effective measures are not taken to control and manage it in time, the seepage accident of tailings pond may be caused.

\subsubsection{Clustering Coefficient and Small-World Property.} According to the definition of clustering coefficient, the node with degree value of 1 has no agglomeration. Therefore, when calculating the clustering coefficient of each node in the PNSRTP, the node with degree value of 1 is excluded. The clustering coefficient of each point in the PNSRTP is shown in Figure 6. It can be seen from Figure 6 that the clustering coefficient of nodes is between 0 and 0.5. The clustering efficiency of hazards 83 (the rising speed of tailings between dam downstream and upstream is unbalanced) and 266 (insufficient production capacity of dryer) is 0.5 . The clustering of other most nodes is between 0.05 and 0.35 , and the distribution is more uniform. This reflects the aggregation of the PNSRTP around hazards 83 and 266.

In order to determine whether the PNSRTP has the small-world property, this paper uses Pajek complex network software to generate a random network with the same number of nodes and average degree value as the PNSRTP, as shown in Figure 7. After calculation, the average clustering coefficient of this random network is 0.04 , which is far less than that of the PNSRTP (0.19). Combined with the fact that the average path length of the PNSRTP is less than 5, it can be concluded that the PNSRTP has the small-world property [32].

\section{Importance and Treatment Sequence of Hazard Nodes}

The global network efficiency of seepage network refers to the average value of the reciprocal sum of the shortest path lengths between pairs of hazard nodes, which reflects the propagation speed of seepage risk in the PNSRTP $[33,34]$. According to the above definition, this paper takes the global network efficiency as an index to measure the management effect of seepage risk after the treatment of seepage hazard.

The betweenness centrality and the degree value and the node clustering coefficient mentioned above are the indicators to measure the importance of nodes. In the process of seepage risk propagation, the node with larger betweenness centrality is the main channel of risk propagation. Therefore, in the treatment of tailings dam seepage risk, we should focus on the nodes with larger betweenness centrality. After calculation, the overall betweenness centralization of the PNSRTP is 0.0643 and the betweenness centrality of each node is shown in Figure 8. It can be seen from Figure 8 that the distribution of the betweenness centrality of the nodes presents serious heterogeneity and the betweenness centrality among the nodes has serious uneven distribution. The betweenness centrality of hazards 65 (dam deformation), 267 (pipes and grooves deformation), and 253 (concentration equipment failure) is $0.0669,0.0647$, and 0.0577 , respectively, which are significantly larger than those of other nodes, indicating that timely treatment of these hazards is helpful to reduce the spread of seepage risk of tailings dam.

In order to verify which one of the three indicators can reduce the global efficiency of the network more quickly and effectively, that is, to reduce the spread of seepage risk, according to the order (size) of the index value, the hazard nodes are treated (deleted) in turn in this paper and then the global efficiency of the network after treatment is calculated. In the management of hazard nodes, the hazard node with the largest index value is treated for the first time and then 5 hazards are treated at a time according to the order of index values, until all hazards are treated. The decline of the network efficiency of the PNSRTP under the three hazard remediation methods is shown in Figure 9.

As can be seen from Figure 9, after the hazard of high clustering coefficient is treated, the network efficiency cannot decline rapidly. Even when the node deletion ratio is less than about $70 \%$, the network efficiency shows an increasing trend. Therefore, the nodes with a large clustering coefficient are not suitable for priority governance. Both betweenness centrality and degree centrality can quickly reduce the network efficiency of the PNSRTP, but obviously, the effect of degree centrality is better. When the ratio of hazard treatment is less than 5\%, the two indexes have the same effect on the reduction of seepage risk and even the betweenness centrality is slightly dominant. However, with the increase in remediation proportion, the gap between the two began to increase. When the governance ratio reaches about $30 \%$, the network efficiency of the PNSRTP is quickly reduced to around 0 under the degree centrality, while the network efficiency is maintained between 0.1 and 0.15 under the betweenness centrality. Therefore, in the prevention and control of tailings pond seepage risk, we should select degree centrality as the index to measure the importance of nodes and give priority to the nodes with large degree value.

\section{Discussion}

The cause of seepage accident in tailings pond is complex, and the seepage hazards changes with the life cycle of tailings ponds, which makes it very difficult to identify the seepage hazard completely and accurately. At the same time, the existing methods of hazard identification are mainly based on the subjective judgment of researchers and these methods lack objective supporting evidence. In order to solve these problems, this paper divides the seepage system of tailings pond into eight subsystems according to its functions and composition characteristics and identifies the seepage hazards in different life cycles according to laws and regulations, accident cases, and other evidence. The evidence is collectively compiled by the most experienced and knowledgeable experts in the industry. At the completion of the compilation, it has undergone decades of practical application verification. After multiple rounds of updates and improvements, the evidence can fully meet the goal of 


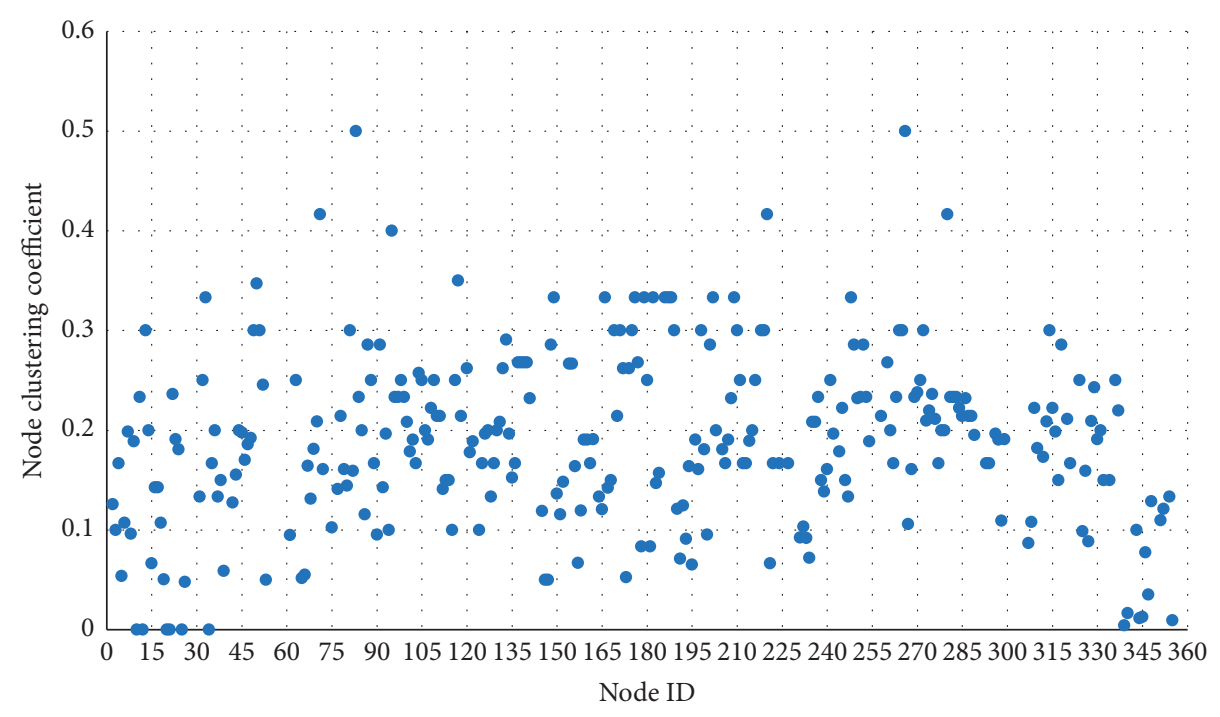

Figure 6: Clustering coefficient of nodes in PNSRTP.

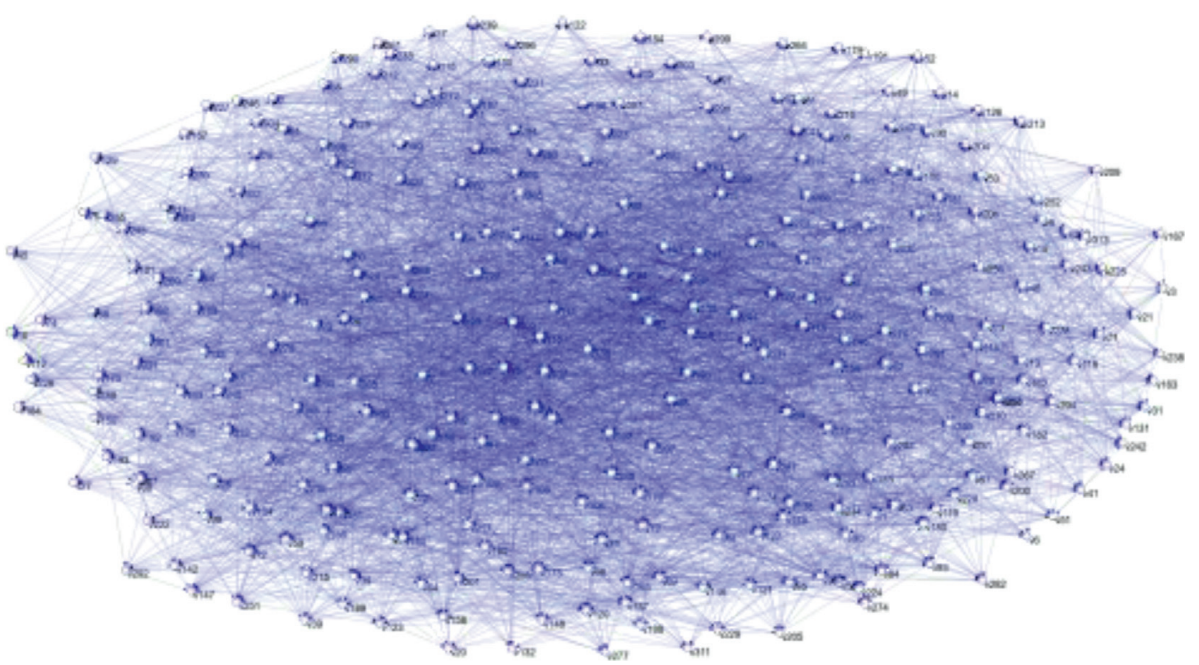

FIgURE 7: Equal-sized seepage risk random network.

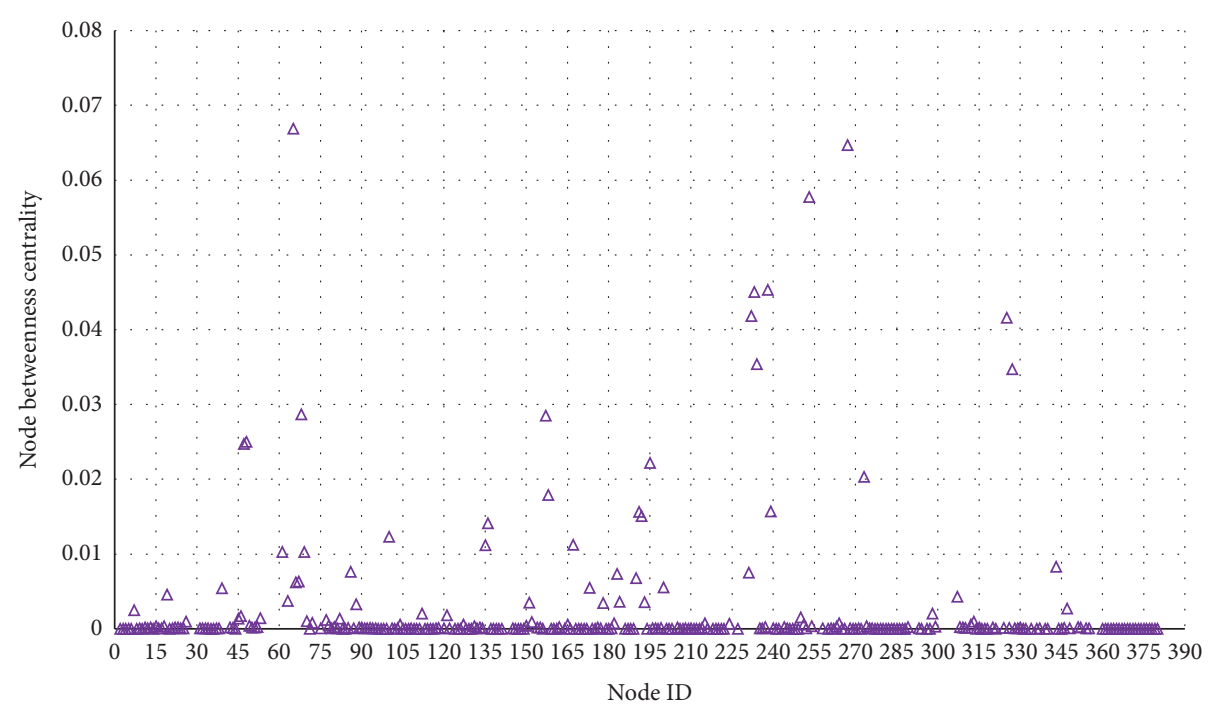

Figure 8: Betweenness centrality of nodes in the PNSRTP. 


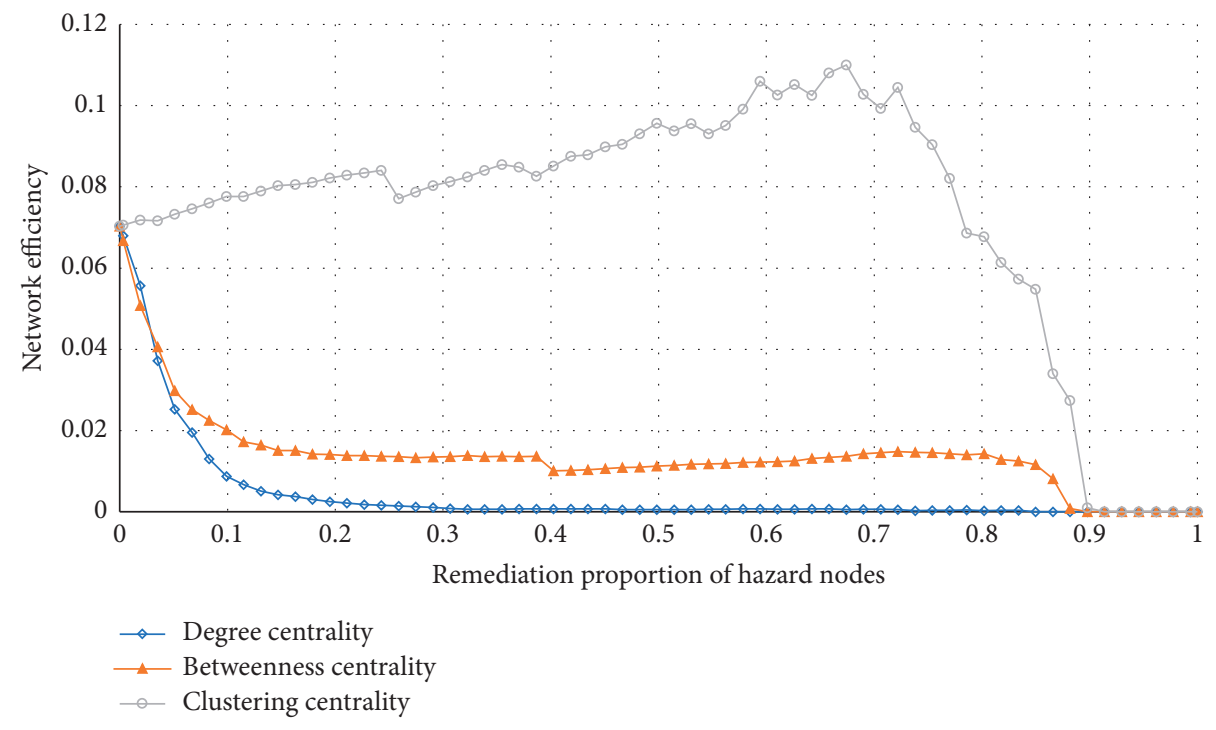

Figure 9: The change of the network efficiency of the PNSRTP.

identifying all the hazards of the seepage system [29]. In a sense, the EIMTSH is a separation of the causal model, which divides the complex system and various influencing factors into small modules to reduce the difficulty of hazard identification.

The nodes and edges of the complex network can better represent the operation process of the complex system. Therefore, this paper takes the seepage hazards as the nodes and the relationship between hazards as the edges to represent the propagation process of seepage risk of tailings pond and constructs the PNSRTP. By analyzing the network characteristics of the PNSRTP with universal applicability, we can get the following: (a) The cumulative degree distribution of the PNSRTP presents a power-law distribution, which shows that the PNSRTP presents the scale-free property. (b) The PNSRTP has a larger clustering coefficient and smaller characteristic path length, which indicates that the network is a small-world network. Compared with the nodes with high betweenness centrality and high clustering coefficient, the nodes with higher degree value can reduce the network efficiency more quickly, which indicates that the nodes with higher degree value have greater importance in the propagation of seepage risk and belong to the hazard nodes with priority governance. It should be noted that the conclusion here is drawn from the perspective of the PNSRTP as a whole. Since the hazards of the tailings ponds and the relationship between hazards are constantly changing in a real tailings pond, it is necessary to use the safety inspection data, work logs, and safety evaluations of the tailings ponds to determine them.

The advantage of the EIMTSH is that it provides support for the complete identification of hazards and the relationship between hazards. However, these support evidence pieces are simplified to have the same reliability without considering the strength of the relationship between hazards, which is obviously different from the actual propagation process of seepage risk. In addition, we analyzed the PNSRTP as a universal network, which contains all the hazards and their relationship of different types and different life cycles of tailings ponds, but the actual operating tailings pond only has some of these hazards and their relationship. This may lead to that some properties and conclusions obtained by analyzing the universal PNSRTP are not applicable to the actual seepage system of tailings ponds.

In order to solve the above problems, the authors will consider the classification of supporting evidence in the future and divide the relationship weight between hazards by combining with AHP, triangular fuzzy, and other methods. At the same time, a number of specific cases are selected to use the above methods for application analysis and research, the nature differences between the PNSRTP and the case networks are observed, and then the above methods are modified and improved, so as to build a more practical research system for the seepage risk of tailings ponds.

\section{Conclusion}

This paper uses the proposed EIMTSH to identify the hazards of the tailings pond seepage system and obtains a hazard list supported by evidence. This list includes a total of 313 hazards and 1912 relationships among hazards. This list will help decision makers and scientists to actively participate in the evidence-based risk assessment process for tailing pond seepage.

Based on the identified list of seepage hazards in tailings ponds, the PNSRTP with nodes of three layers and two stages is constructed based on the complex network theory, which realizes the characterization of the seepage risk propagation process of tailings pond. Through the analysis of the characteristics of the PNSRTP, we can find that the PNSRTP has the scale-free property and small-world property.

In this paper, the network efficiency is selected as the criterion of seepage risk propagation ability. By comparing the effects of degree centrality, node betweenness centrality, and clustering coefficient in reducing the network efficiency 
of the PNSRTP, it is concluded that nodes with higher degree values play a more important role in the process of seepage risk propagation. Through giving priority to the treatment of nodes with a high degree value, the seepage risk can be reduced more quickly and the occurrence of seepage accidents can be avoided, which is conducive to the sustainable development of the mining industry.

\section{Appendix}

\section{A. Laws, Standards, and Norms of Supporting Evidence}

\begin{tabular}{lc}
\hline Code & Name \\
\hline F1 & Code for Design of Tailings Facilities (GB 50863-2013) \\
F2 & Code for Construction and Acceptance of Tailings \\
F3 & Facilities (GB-T 50864-2013) \\
F4 & Safety Regulations for Tailings Pond (GB GB39496-2020) \\
F5 & Code for Construction of Tailings Facilities (AQ \\
& 2001-2018) \\
F6 & $\begin{array}{c}\text { Determination of Hidden Dangers of Major Production } \\
\text { Safety Accidents in Metal And Non-Metal Mines }\end{array}$ \\
& Standards (trial) (safety supervisor no. 1 [2017] no. 98) \\
\hline
\end{tabular}

\section{B. Scientific Literature Supporting Evidence}

\begin{tabular}{|c|c|}
\hline Code & Literature \\
\hline W1 & $\begin{array}{c}\text { Wang Yishui, Peng Zeng, Xiao Chuibin. Selections of Mine } \\
\text { Geology. Volume Seven, Technical Manual of Tailings } \\
\text { Pond Design, Construction, Management and } \\
\text { Development and Utilization of Tailings Resources. } \\
\text { Central South University Press, 2015. }\end{array}$ \\
\hline W2 & $\begin{array}{c}\text { Zhao Yiqing. Representation theory and model of hazards } \\
\text { and risks of tailings ponds[M]. Metallurgical Industry } \\
\text { Press, } 2016 .\end{array}$ \\
\hline W3 & $\begin{array}{r}\text { Qin Xuan, Li Zhongxue, Zh } \\
\text { model of tailing pond risk e } \\
\text { hazards[J]. Systems Engine }\end{array}$ \\
\hline W4 & $\begin{array}{l}\text { Zhao Yiqing, Qin Xuan, Li Zhongxue, et al. System } \\
\text { dynamics simulation and simulation of hazards and risk } \\
\text { evolution of tailings ponds[J]. Journal of University of } \\
\text { Science and Technology Beijing, 2014(9):1158-1165. }\end{array}$ \\
\hline W5 & $\begin{array}{l}\text { Recognition of hazards of tailing pond acc } \\
\text { process-cause grid method[J]. China Wor } \\
\text { and Technology, 2013, 9(004):9 }\end{array}$ \\
\hline W6 & $\begin{array}{c}\text { Li Quanming, Wang Yunhai, Zhang Xingkai, et al. Analysis } \\
\text { of dam-break disaster factors of tailing pond and research } \\
\text { on risk index system[J]. China Work Safety Science and } \\
\text { Technology, 2008(03):50-53. }\end{array}$ \\
\hline W7 & $\begin{array}{l}\text { Liu Haiming, Cao Jing, Yang Chunhe. Analysis of disaster- } \\
\text { causing factors of tailing dam accidents at home and } \\
\text { abroad[J]. Metal Mine, 2013, 42(2):126-129. }\end{array}$ \\
\hline
\end{tabular}

TABLE : Continued.

\begin{tabular}{|c|c|}
\hline Code & Literature \\
\hline W8 & $\begin{array}{l}\text { Chai Jianshe, Wang Shu, Men Yongsheng. Case analysis } \\
\text { and accident prediction of tailing pond accidents[M]. } \\
\text { Chemical Industry Press, } 2011 \text {. }\end{array}$ \\
\hline W9 & $\begin{array}{c}\text { M. Rico, Benito G, Salgueiro A R, et al. Reported tailings } \\
\text { dam failures: A review of the European incidents in the } \\
\text { worldwide context[J]. Journal of Hazardous Materials, } \\
2008 \text {. }\end{array}$ \\
\hline W10 & $\begin{array}{l}\text { Hatje V, Pedreira R M A, De Rezende C E, et al. The } \\
\text { environmental impacts of one of the largest tailing dam } \\
\text { failures worldwide[J]. entific Reports, } 2017,7(1): 10706 \text {. } \\
\text { RISKGATE }\end{array}$ \\
\hline W11 & $\begin{array}{c}\text { RISKGATE is an Australian coal industry initiative driven } \\
\text { by The University of Queensland, ACARP, and industry } \\
\text { partners ; }\end{array}$ \\
\hline W12 & $\begin{array}{c}\text { Assessing Risks of Mine Tailing Dam Failures, Paulina } \\
\text { Concha Larrauri, August, } 2017\end{array}$ \\
\hline
\end{tabular}

\section{Accident Case Supporting Evidence}

\begin{tabular}{|c|c|c|c|c|}
\hline Code & Date & Location & $\begin{array}{l}\text { Type of } \\
\text { incident }\end{array}$ & Impacts \\
\hline $\mathrm{C} 1$ & $\begin{array}{l}2020 \\
\text { Mar. } \\
28\end{array}$ & $\begin{array}{l}\text { Tieli, Yichun City, } \\
\text { Heilongjiang } \\
\text { Province, China }\end{array}$ & $\begin{array}{l}\text { Seepage or } \\
\text { leakage }\end{array}$ & $\begin{array}{l}\text { Water and tailings } \\
\text { flowed through } \\
\text { surrounding area, } \\
\text { reaching Yijimi } \\
\text { River after } 3 \mathrm{~km} \text {, } \\
\text { threatening the } \\
\text { drinking water } \\
\text { resource of } 68,000 \\
\text { people in Tieli } \\
\text { City; by Apr. } 4 \text {, the } \\
\text { pollution reached } \\
208 \mathrm{~km} \\
\text { downstream }\end{array}$ \\
\hline C2 & $\begin{array}{l}2019 \\
\text { Jan. } \\
25\end{array}$ & $\begin{array}{c}\text { Córrego de Feijão } \\
\text { Mine, Brazil }\end{array}$ & $\begin{array}{l}\text { Tailings dam } \\
\text { failure and } \\
\text { seepage }\end{array}$ & $\begin{array}{l}259 \text { people were } \\
\text { killed, and } 11 \text { are } \\
\text { reported missing } \\
\text { The toxic }\end{array}$ \\
\hline C3 & $\begin{array}{c}2017, \\
\text { June } \\
30\end{array}$ & $\begin{array}{l}\text { Mishor Rotem, } \\
\text { Israel }\end{array}$ & $\begin{array}{l}\text { Tailings dam } \\
\text { failure, } \\
\text { overtopping, } \\
\text { and seepage }\end{array}$ & $\begin{array}{l}\text { wastewater surged } \\
\text { through the dry } \\
\text { Ashalim riverbed } \\
\text { and left a wake of } \\
\text { ecological } \\
\text { destruction more } \\
\text { than } 20 \mathrm{~km} \text { long }\end{array}$ \\
\hline C4 & $\begin{array}{l}2016, \\
\text { Oct. } \\
27\end{array}$ & $\begin{array}{c}\text { Antamok Mine } \\
\text { (inactive), Itogon, } \\
\text { Benguet Province, } \\
\text { Philippines }\end{array}$ & $\begin{array}{l}\text { Seepage or } \\
\text { leakage }\end{array}$ & $\begin{array}{c}\text { The leaked tailings } \\
\text { flowed into Liang } \\
\text { River, then } \\
\text { Ambalanga River } \\
\text { before reaching } \\
\text { Agno River } \\
\text { Thee leaked }\end{array}$ \\
\hline C5 & $\begin{array}{l}2016 \\
\text { Aug. } \\
27\end{array}$ & $\begin{array}{c}\text { New Wales Plant, } \\
\text { Mulberry, Polk } \\
\text { County, Florida, } \\
\text { USA }\end{array}$ & $\begin{array}{l}\text { Seepage or } \\
\text { leakage }\end{array}$ & $\begin{array}{l}\text { tailings flowed } \\
\text { into Liang River, } \\
\text { then Ambalanga } \\
\text { River before } \\
\text { reaching Agno } \\
\text { River }\end{array}$ \\
\hline
\end{tabular}


TABle : Continued.

\begin{tabular}{|c|c|c|c|c|}
\hline Code & Date & Location & $\begin{array}{l}\text { Type of } \\
\text { incident }\end{array}$ & Impacts \\
\hline C6 & $\begin{array}{l}2012, \\
\text { Nov. } 4\end{array}$ & $\begin{array}{l}\text { Sotkamo, Kainuu } \\
\text { Province, Finland }\end{array}$ & $\begin{array}{l}\text { Seepage or } \\
\text { leakage }\end{array}$ & $\begin{array}{l}\text { Nickel and zinc } \\
\text { concentrations in } \\
\text { nearby snow river } \\
\text { exceeded the } \\
\text { values that are } \\
\text { harmful to } \\
\text { organisms tenfold } \\
\text { or even a } \\
\text { hundredfold, } \\
\text { uranium } \\
\text { concentrations } \\
\text { more than tenfold }\end{array}$ \\
\hline C7 & $\begin{array}{l}2004 \\
\text { May } \\
22\end{array}$ & $\begin{array}{c}\text { Partizansk, } \\
\text { Primorski Krai, } \\
\text { Russia }\end{array}$ & $\begin{array}{l}\text { Seepage or } \\
\text { leakage }\end{array}$ & $\begin{array}{l}\text { The ash flowed } \\
\text { through a drainage } \\
\text { canal into a } \\
\text { tributary to the } \\
\text { Partizanskaya } \\
\text { River which } \\
\text { empties in to } \\
\text { Nahodka Bay in } \\
\text { Primorski Krai } \\
\text { (east of } \\
\text { Vladivostok) }\end{array}$ \\
\hline C8 & $\begin{array}{l}\text { 1996, } \\
\text { Mar. } \\
24\end{array}$ & $\begin{array}{c}\text { Marcopper, } \\
\text { Marinduque Island, } \\
\text { Philippines }\end{array}$ & $\begin{array}{l}\text { Seepage or } \\
\text { leakage }\end{array}$ & $\begin{array}{l}\text { Evacuation of } \\
1200 \text { residents, } \\
18 \mathrm{~km} \text { of river } \\
\text { channel filled with } \\
\text { tailings, US\$ } 80 \\
\text { million damage }\end{array}$ \\
\hline C9 & $\begin{array}{l}\text { 1994, } \\
\text { June }\end{array}$ & $\begin{array}{c}\text { Sinkhole Opens in } \\
\text { Phosphogypsum } \\
\text { Stake }\end{array}$ & $\begin{array}{l}\text { Seepage or } \\
\text { leakage }\end{array}$ & $\begin{array}{l}\text { Release of gypsum } \\
\text { and water into } \\
\text { groundwater }\end{array}$ \\
\hline $\mathrm{C} 10$ & $\begin{array}{c}1994 \\
\text { Feb. } \\
14\end{array}$ & $\begin{array}{l}\text { Olympic Dam, } \\
\text { Roxby Downs, } \\
\text { South Australia }\end{array}$ & $\begin{array}{l}\text { Seepage or } \\
\text { leakage }\end{array}$ & $\begin{array}{l}\text { Leakage of tailings } \\
\text { dam during } 2 \\
\text { years or more }\end{array}$ \\
\hline $\mathrm{C} 11$ & 1993 & Marsa, Peru & $\begin{array}{l}\text { Dam failure, } \\
\text { overtopping, } \\
\text { and seepage }\end{array}$ & 6 people killed \\
\hline $\mathrm{C} 12$ & 1986 & $\begin{array}{l}\text { Huangmeishan, } \\
\text { China }\end{array}$ & $\begin{array}{l}\text { Dam failure } \\
\text { and seepage }\end{array}$ & 19 people killed \\
\hline $\mathrm{C} 13$ & $\begin{array}{l}\text { 1966, } \\
\text { May } 1\end{array}$ & $\begin{array}{l}\text { Mir mine, } \\
\text { Sgorigrad, Bulgaria }\end{array}$ & $\begin{array}{l}\text { Dam failure } \\
\text { and seepage }\end{array}$ & $\begin{array}{l}\text { Dam failure from } \\
\text { rising pond level } \\
\text { after heavy rains } \\
\text { and/or failure of } \\
\text { diversion channel }\end{array}$ \\
\hline
\end{tabular}

\section{Data Availability}

The data that support the findings of this study are included within the paper.

\section{Conflicts of Interest}

The authors declare that they have no conflicts of interest.

\section{Acknowledgments}

This work was supported by the National Key Research and Development Program of China (Grant no. 2017YFC0804605).

\section{References}

[1] Y. Wang, P. Zeng, and C. Xiao, Selections of Mine Geology. Volume Seven, Technical Manual of Tailings Pond Design, Construction, Management and Tailings Resources Development and Utilization, Central South University Press, Changsha, 2015.

[2] M. Rico, G. Benito, A. R. Salgueiro, A. Díez-Herrero, and H. G. Pereira, "Reported tailings dam failures," Journal of Hazardous Materials, vol. 152, no. 2, pp. 846-852, 2008.

[3] X. Qin, Z. Li, and Y. Zhao, "Complex network model of tailing pond risk evolution and analysis of key hidden dangers," Systems Engineering Theory and Practice, vol. 37, no. 6, 2017.

[4] S. Tong, W. Zheng, and B. Chen, "Analysis of pollution consequences of toxic liquid leakage and see page," Industrial Safety and Environmental Protection, vol. 2006, no. 10, pp. 59-61, 2006.

[5] S. Shu, W. Zhu, and J. Shi, "A new simplified method to calculate breakthrough time of municipal solid waste landfill liners," Journal of Cleaner Production, vol. 219, no. 10, pp. 649-654, 2019.

[6] X. He, C. Zheng, X. Sui et al., "Biological damage to SpragueDawley rats by excessive anions contaminated groundwater from rare earth metals tailings pond seepage," Journal of Cleaner Production, vol. 185, no. 1, pp. 523-532, 2018.

[7] C. A. López-Morales and R. T. Lilia, "On the economic analysis of wastewater treatment and reuse for designing strategies for water sustainability: lessons from the Mexico Valley Basin," Resources, Conservation and Recycling, vol. 140, pp. 1-12, 2018.

[8] ISO 31000, Risk Management Guide, ISO, Geneva, Switzerland, 2018.

[9] Z. Li, X. Deng, and Y. Zhang, "Evaluation and convergence analysis of socio-economic vulnerability to natural hazards of belt and road initiative countries," Journal of Cleaner Production, vol. 282, no. 5, Article ID 125406, 2021.

[10] P. Makowski and Z. Niedbalski, "A comprehensive geomechanical method for the assessment of rock burst hazards in underground mining," International Journal of Mining Science and Technology, vol. 30, no. 3, 2020.

[11] M. A. Ibrahim, "Risk of spontaneous and anthropogenic fires in waste management chain and hazards of secondary fires," Resources, Conservation and Recycling, vol. 159, 2020.

[12] C. Ferreira, J. Ribeiro, and F. Freire, "A hazard classification system based on incorporation of REACH regulation thresholds in the USEtox method," Journal of Cleaner Production, vol. 228, no. 10, pp. 856-866, 2019.

[13] W. Deng, J. Xu, Y. Song, and H. Zhao, "Differential evolution algorithm with wavelet basis function and optimal mutation strategy for complex optimization problem," Applied Soft Computing, vol. 100, Article ID 106724, 2021.

[14] W. Deng, J. Xu, X.-Z. Gao, and H. Zhao, "An enhanced MSIQDE algorithm with novel multiple strategies for global optimization problems," IEEE Transactions on Systems, Man, and Cybernetics: Systems, pp. 1-10, 2020.

[15] W. Deng, J. Xu, H. Zhao, and Y. Song, "A novel gate resource allocation method using improved PSO-based QEA," IEEE Transactions on Intelligent Transportation Systems, pp. 1-9, 2020.

[16] Z. Li, Identification and Control of Disaster-Causing Factors in Tailing Ponds, Northeastern University, Shenyang, 2008. 
[17] S. Gao, Y. Xu, G. Zhao, and M. Wang, "Numerical simulation of seepage flow in dikes based on detection of hidden dangers of ground penetrating radar," People's Yangtze River, vol. 48, no. S1, pp. 158-161, 2017.

[18] Z. Wang, Research on Seepage Monitoring Method of EarthRock Embankment Based on Distributed Optical Fiber Temperature Measurement Technology, Xi'an University of Technology, Xi'an, 2019.

[19] Q. Ma, A. Zhang, and J. Sun, "Treatment of hidden dangers of seepage safety in the main dam of Yuecheng Reservoir," Haihe Water Resources, vol. 2011, no. 5, pp. 19-21, 2011.

[20] S. Nan, Y. Jia, X. Zhao, S. L. Ho, and C. W. Kan, “A complex network based model for detecting isolated communities in water distribution networks," Chaos: An interdisciplinary journal of nonlinear science, vol. 23, no. 4, pp. 3323-3335, 2013.

[21] X. Wang, W. Wei, J. Ge et al., "Embodied rare earths flow between industrial sectors in China: a complex network approach," Resources, Conservation and Recycling, vol. 125, pp. 363-374, 2017.

[22] J. Shi, C. Zhang, and W.-Q. Chen, "The expansion and shrinkage of the international trade network of plastic wastes affected by China's waste management policies," Sustainable Production and Consumption, vol. 25, pp. 187-197, 2021.

[23] S. Guo, X. Zhou, B. Tang, and P. Gong, "Exploring the behavioral risk chains of accidents using complex network theory in the construction industry," Physica A: Statistical Mechanics and Its Applications, vol. 560, 2020.

[24] Y. T. Mohmand and A. Wang, "Complex network analysis of Pakistan railways," Discrete Dynamics in Nature and Society, vol. 2014, Article ID 126261, 5 pages, 2014.

[25] E.-Y. Yu, Y.-P. Wang, Y. Fu, D.-B. Chen, and M. Xie, "Identifying critical nodes in complex networks via graph convolutional networks," Knowledge-Based Systems, vol. 198, Article ID 105893, 2020.

[26] L. Zhiwei, Z. A. Nan, X. B. Fei, and N. Chen, "A novel measure of identifying influential nodes in complex networks," Physica A: Statistical Mechanics and Its Applications, vol. 523, pp. 488-497, 2019.

[27] Z. Zhen, J. Li, and Z. Li, "Hazard identification and risk decision-making system of tailings storage facilities based on complex networks," in Proceedings of the 2020 IEEE 9th Joint International Information Technology and Artificial Intelligence Conference (ITAIC), December 2020.

[28] M. Dadvar, Hazard Definition and Classification Review, UNDDR, Geneva, Switzerland, 2020.

[29] Z. Zhen, J. Li, and Z. Li, "Exploration and research on the propagation law of failure risk of tailings dam based on complex networks," Physica A: Statistical Mechanics and Its Applications, In press, 2021.

[30] F. A. C. Nascimento, A. Majumdar, W. Y. Ochieng, and S. R. Jarvis, "A multistage multinational triangulation approach to hazard identification in night-time offshore helicopter operations," Reliability Engineering \& System Safety, vol. 108, pp. 142-153, 2012.

[31] Z. Zhen, B. Ma, and H. Zhao, "Propagation network of tailings dam failure risk: an empirical research and the identification of key hazard node," 2021. Available from: https://www. researchsquare.com/article/rs-778838/v1.

[32] D. J. Watts and S. H. Strogatz, "Collective dynamics of 'smallworld' networks,” Nature, vol. 393, no. 6684, pp. 440-442, 1998.
[33] V. Latora and M. Marchiori, "Efficient behavior of smallworld networks," Physical Review Letters, vol. 87, no. 19, Article ID 198701, 2001.

[34] V. Latora and M. Marchiori, "Economic small-world behavior in weighted networks," The European Physical Journal B-Condensed Matter and Complex Systems, vol. 32, no. 2, pp. 249-263, 2003. 